\title{
Comparação do efeito agudo do exercício com a eletroestimulação funcional sobre o fluxo arterial periférico de indíviduos hipertensos e diabéticos: um estudo piloto
}

\author{
comparison of the acute effect of exercise with the \\ electrostimulation functional on the peripheral artery flow \\ of diabetic and hypertensive individuals: a pilot study
}

Djeime Mahle, ${ }^{1}$ Taís Borges, ${ }^{1}$ Marciele Silveira Hopp, ${ }^{1}$ Litiele Evelin Wagner, ${ }^{1}$ Bárbara da Costa Flores, Dulciane Nunes Paiva, ${ }^{2}$ Dannuey Machado Cardoso ${ }^{1,2}$

'Universidade de Santa Cruz do Sul (Unisc), Santa Cruz do Sul, RS, Brasil.

2Universidade Federal do Rio Grande do Sul (UFRGS), Porto Alegre, RS, Brasil.

Recebido em: 19/07/2016 / Aceito em: 22/08/2016 / Publicado em: 18/10/2016

dannueyc@unisc.br

\section{RESUMO}

Objetivo: comparar o efeito agudo do exercício de planti/dorsi flexão de tornozelo com a eletroestimulação funcional (FES) sobre o fluxo arterial periférico de indivíduos com Hipertensão Arterial Sistêmica (HAS) e Diabetes Mellitus (DM) tipo II. Método: estudo crossover composto por 10 participantes portadores de HAS e DM controlada, sendo 5 do sexo masculino, com média de idade de $61,6 \pm 8,1$ anos e IMC de 29,7 $\pm 3,7 \mathrm{Kg} / \mathrm{m}^{2}$ Avaliado o Índice Tornozelo-Braquial (ITB) em membros superiores e inferiores por meio de esfigmomanômetro e doppler vascular portátil, sendo avaliada a presença de claudicação intermitente (CI), por meio do Questionário de Edimburgo em repouso. Após, a amostra foi randomizada para receber a aplicação da FES (situação intervenção) ou realizar exercício de planti/dorsiflexão de tornozelo (situação controle) com aferição posterior do ITB, sendo conferida pausa entre intervenções (washout) de 10 a 20 segundos. Resultados: Na amostra avaliada, dois participantes apresentavam ITB com indicativo de doença arterial periférica (DAP) $(<0,90)$ estando a $\mathrm{Cl}$ presente em oito indivíduos da amostra. Tanto o exercício quanto a FES não produziram diferença significativa no ITB, entretanto, a FES produziu um tamanho do efeito médio a grande, indicando redução aguda do fluxo periférico. Considerações finais: $\mathrm{Na}$ amostra estudada, a FES e exercício ativo de plati/ dorsiflexão de tornozelo não alteraram o fluxo arterial periférico de indivíduos com HAS e DM tipo II, no entanto, devido à tendência deste índice ser menor após aplicada a FES, o exercício parece ser mais seguro para este perfil de paciente.

Palavras-chave: Diabetes Mellitus; Hipertensão; Eletroestimulação muscular; Índice Tornozelo Braquial; Doença Arterial Obstrutiva Periférica.

\section{ABSTRACT}

Objective: comparing the acute effect of exercise of plati/dorsi flexion ankle with functional electrostimulation (FES) on the peripheral artery flow of patients with systemic arterial hypertension (SAH) and diabetes mellitus (DM) type II. Method: Crossover study composed of 10 participating patients with SAH and DM controlled, 5 males, mean age $61.6 \pm 8.1$ years and BMI $29.7 \pm 3.7 \mathrm{Kg} / \mathrm{m}^{2}$. Ankle-Brachial Index (ABI) reviewed in upper and lower limbs through sphygmomanometer and portable doppler vascular being evaluated the presence of intermittent claudication (IC) through the Edinburgh questionnaire in repose. After, the sample was randomized to receive the application of FES (intervention situation) or perform exercise planti) dorsiflexion of ankle (control situation) with subsequent measurement of $A B I$, being given pause between interventions (washout) of 10 to 20 seconds. Results: in the sample evaluated, two participants had with ITB two 
participants being with ITB indicative of arterial peripheral disease $(P A D)(<0.90)$ being the $C /$ present in 08 Individuals of the sample. Both exercise and FES did not produce significant difference in $A B I$, however, the FES produced a size effect medium to large, indicating acute reduction in peripheral blood flow. Closing remarcks: In the sample studied, the FES and active exercise of plati/dorsiflexion of ankle did not affect the peripheral artery stream of subjects with hypertension and diabetes mellitus type II, however, due to the tendency of this index is lower after applied to FES, exercise seems be safer for this patient profile.

Keywords: Diabetes Mellitus; Hypertension; Muscle electrostimulation; Ankle brachial-index; Peripheral occlusive arterial disease.

\section{INTRODUÇÃO}

A Hipertensão Arterial Sistêmica (HAS) é uma condição clínica multifatorial caracterizada por níveis elevados e sustentados de pressão arterial (PA). Associa-se frequentemente a alterações funcionais e/ ou estruturais dos órgãos-alvo e às alterações metabólicas, com consequente aumento do risco de eventos cardiovasculares fatais e não fatais.

A Diabetes Mellitus (DM) tipo II é uma enfermidade crônica de etiologia múltipla e prevalência mundial, pertencente a um grupo de distúrbios metabólicos que apresenta hiperglicemia comum, sendo uma doença adquirida, que ao longo do tempo, passa por estágios de glicemia de jejum alterada e tolerância à glicose diminuída. ${ }^{2}$

Os fatores de risco para desenvolvimento da HAS e DM tipo II assemelham-se muito com os responsáveis pelo surgimento da doença arterial periférica (DAP), incluindo hábitos modificáveis relacionados ao estilo de vida como tabagismo, dislipidemias, obesidade, sedentarismo, uso abusivo de álcool e características não modificáveis, como idade, sexo e história familiar. Tanto a HAS, como a DM, constituem os principais fatores de risco para as doenças do aparelho circulatório, sendo considerada a primeira causa de morte no Brasil nas últimas décadas. ${ }^{3}$

A Doença Arterial Periférica (DAP) caracteriza-se por ser uma doença de natureza estenosante total ou parcial do lúmen arterial que resulta em déficit de fluxo sanguíneo aos tecidos, com a presença de sinais e sintomas característicos de isquemia, sendo mais prevalente nos membros inferiores. Em cerca de $90 \%$ dos casos da doença, o componente obstrutivo se encontra associado a fenômenos exclusivos de aterosclerose. ${ }^{4}$

Dentre as manifestações desencadeadas pela DAP, a claudicação intermitente $(\mathrm{Cl})$ é a mais usual e se manifesta de forma localizada com geração de dor, cãibra, parestesia ou desconforto muscular. ${ }^{5,6} \mathrm{~A} \mathrm{Cl}$ configura-se em um sinal clínico, que pode indicar insuficiência vascular periférica, sendo o índice tornozelo-braquial (ITB), um método não-invasivo utilizado para detecção de doenças vasculares periféricas através da medida da pressão arterial braquial, pediosa e tibial. Tal índice é considerado anormal quando, a relação entre a maior medida das pressões sistólicas braquial, dividida pela pressão das artérias pediosas e tibial posterior for inferior ou igual a
0,9 ou superior a $1,4 \mathrm{mmHg}$ respectivamente. ${ }^{7}$

A eletroestimulação funcional (FES) é aplicada na prática clínica para promover o fortalecimento de músculos enfraquecidos, bem como, para recuperar ou preservar sua função durante o período de atividade reduzida ou de imobilização. ${ }^{8}$ Tsang et al. $^{9}$ evidenciam que o tratamento com a eletroestimulação deve ser realizado por longos períodos, visto que as mudanças provocadas são temporárias e segundo Medeiros et al., ${ }^{10}$ a FES é um método terapêutico capaz de induzir atividade muscular com aumento da oferta de oxigênio para o músculo, através da redistribuição do fluxo sanguíneo, ocasionando portanto, melhora da perfusão capilar, do consumo de oxigênio e redução da fadigabilidade muscular em pacientes com $\mathrm{Cl}$. No entanto, para nosso conhecimento, não há na literatura estudos que demonstrem o efeito desta corrente elétrica sobre o fluxo arterial periférico, sendo esta importante para avaliar a segurança da aplicação desta terapêutica. Nossa hipótese é de que a FES, assim como o exercício ativo de tornozelo, pode reduzir o fluxo arterial periférico, sendo importante sua avaliação previamente a aplicação da FES. Dessa forma, o objetivo do presente estudo, foi comparar o efeito agudo da FES e do exercício ativo de tornozelo sobre o fluxo arterial periférico em portadores de DM tipo II e HAS.

\section{MÉTODO}

Trata-se de estudo crossover em que a amostra recebeu intervenção com FES e exercícios de dorsi/ plantiflexão de tornozelo bilateralmente de modo randomizado, sendo o paciente, o seu próprio controle. A amostra foi por conveniência, na qual foram avaliados 10 indivíduos hipertensos e diabéticos de uma Estratégia de Saúde da Família (ESF) do município de Santa Cruz do Sul, Rio Grande do Sul, no período de novembro de 2014 a abril de 2015.

Foram incluídos no estudo indivíduos com idade de 45 a 70 anos, de ambos os sexos, com o diagnóstico de DM não insulino dependentes e HAS de leve a moderada com controle medicamentoso (Pressão Arterial Sistólica [PAS] $<200 \mathrm{mmHg}$ e Pressão Arterial Diastólica $[P A D]<110 \mathrm{mmHg}$ ). Foram excluídos aqueles com déficit de compreensão, lesões dérmicas na região de aplicação dos eletrodos da FES ou com qualquer alteração musculoesquelética que impossibilitasse a realização do protocolo da pesquisa. O estudo foi aprovado pelo Comitê de Ética em Pesquisa em Seres Humanos da Universidade de Santa Cruz do Sul, sob o protocolo número 839.444. Ressalta-se que todos os voluntários concordaram com a pesquisa e assinaram previamente o Termo de Consentimento Livre e Esclarecido.

\section{Procedimento}

Foram avaliadas as características antropométricas (peso, altura e o Índice de Massa Corporal (IMC)), os sinais vitais (frequência cardíaca [FC], PAS e PAD, frequência respiratória [FR]) e a saturação periférica de oxigênio $\left(\mathrm{SpO}_{2}\right)$. Os indivíduos avaliados foram questionados quanto ao uso de medicações, sendo posteriormente aplicado o questionário de Edimburgo seguido da 
avaliação do ITB em repouso. Após a avaliação inicial, os sujeitos foram randomizados para receber a aplicação da FES (situação de intervenção) ou realizar o exercício de planti/dorsiflexão de tornozelo (situação controle). Imediatamente após cada situação (aplicação de FES ou exercício) foi aferido novamente o ITB. Uma pausa entre cada intervenção (washout) de 10 a 20 segundos fol conferida até que o ITB retornasse ao valor de repouso.

\section{Randomização}

A randomização foi realizada através de uma lista de ordem aleatória de alocação, sendo utilizado os números 1 e 2 para designar se os sujeitos iriam iniciar o protocolo do estudo pela aplicação da FES ou realização de exercício de planti/dorsiflexão de tornozelo, respectivamente. Para aleatorização dos números 1 e 2 foi utilizado o software Randon Number Generator (versão 1.77, Segobit Software, EUA).

\section{Avaliação da claudicação}

A avaliação da presença de claudicação foi realizada através do questionário de Edimburgo, composto por inquéritos sobre a presença de desconforto em membros inferiores. O resultado é classificado como positivo (presença de claudicação), caso as respostas às perguntas tenham sido $1=$ "sim", 2 = "não", 3 = "sim", 5 = "geralmente desaparece em 10 minutos ou menos" e 6 = "panturrilha" e/ou "coxa" e/ou "região glútea" ou negativo se assinalada qualquer combinação diferente das descritas acima. ${ }^{11}$

\section{Mensuração do Índice Tornozelo-Braquial}

O fluxo arterial periférico foi avaliado através do ITB, sendo tal variável obtida através da medida da pressão arterial sistólica braquial em ambos os membros superiores e tornozelos, nas artérias pediosa e tibial posterior, conforme recomendação da American Heart Association. ${ }^{12}$ A aferição da PAS nas artérias braquiais, pediosa e tibial posterior bilateralmente foi realizada através de doppler vascular portátil (MEDPEJ ${ }^{\bullet}$ - modelo 2001, Brasil) com esfigmomanômetro posicionado a 03 centímetros acima da fossa cubital e 03 centímetros acima do maléolo medial. Após as medidas, dividiu-se o maior valor entre os $\mathrm{MI}$ (PAS ${ }_{\mathrm{mi}}$ ) pelo maior valor entre os MS (PAS $\left.{ }_{m s}\right)$ para o cálculo do ITB geral $\left(\right.$ ITB $_{\text {geral }}=$ PAS $_{m i} /$ PAS $\left._{\mathrm{ms}}\right) .{ }^{12}$ Classifica-se como um ITB normal os valores de 1.00 a 1.40, limítrofe 0,91 a 0,99 e indicativo de DAP menor que $0.90 .^{13}$

\section{Protocolo para exercício de planti/dorsiflexão de tornozelo}

O protocolo de exercício foi composto por 2,5 minutos de exercícios de planti/dorsiflexão ativa de tornozelo bilateralmente abrangendo 60 repetições $/ \mathrm{min} .{ }^{14}$

\section{Protocolo para estimulação elétrica funcional}

Foram empregues a estimulação dos músculos gastrocnêmios e quadríceps através de eletrodos de silicone de carbono e gel sobre o ventre muscular de ambos os músculos bilateralmente. Os parâmetros de largura do pulso foram de $150 \mu$ s, frequência de $80 \mathrm{~Hz}$, com rampa de sustentação de 3 segundos e repouso de 2 segundos com tempo total de aplicação de 20 min em uma única intervenção. ${ }^{10}$

Para detectar uma diferença significativa no escore do ITB entre o pré e pós exercício com nível de significância de 5\% (bicaudal), poder de $80 \%$ e perda de $10 \%$, o tamanho amostral necessário foi de 15 pacientes. ${ }^{14}$ Os dados foram analisados através do software SPSS (versão 20.0, EUA) e expressos em média e desvio padrão. A normalidade dos dados foi avaliada através do Teste de Shapiro-Wilk, sendo estes transformados em logaritmo natural para obter-se tal normalidade. Para comparar o ITB pré e pós intervenção foi utilizado o teste $t$ de Student pareado e a comparação da variação do ITB entre as intervenções foi realizada através do teste $t$ Student para amostras independentes. Considerando que o poder do estudo é definido como a capacidade de demonstrar uma diferença estatisticamente significativa (ou "efeito"), o tamanho do efeito foi determinado a partir do cálculo d de Cohen, ${ }^{15}$ para comparações entre o efeito do exercício e da FES. Foi considerado significativo um $\mathrm{p}<0,05$.

\section{RESULTADOS}

Foram recrutados para o estudo 24 sujeitos, sendo que 11 não compareceram no momento da avaliação e 3 foram excluídos por apresentarem lesões de pele na região de aplicação dos eletrodos, o que totalizou a amostra final de 10 indivíduos. Na Tabela 1 evidenciam-se as características antropométricas e clínicas da amostra.

Tabela 1 - Características da amostra.

\begin{tabular}{|c|c|}
\hline Variáveis & $(n=10)$ \\
\hline Sexo masculino, n (\%) & $5(50)$ \\
\hline Idade (anos) & $61,6 \pm 8,1$ \\
\hline Peso (Kg) & $80,5 \pm 13,0$ \\
\hline Altura (cm) & $164,3 \pm 7,5$ \\
\hline IMC (Kg/m2) & $29,7 \pm 3,7$ \\
\hline Tabagismo ativo, n (\%) & $2(20)$ \\
\hline Ex-tabagista, n (\%) & $1(10)$ \\
\hline Claudicação, n (\%) & $8(80)$ \\
\hline \multicolumn{2}{|l|}{ Sinais vitais } \\
\hline FC (bpm) & $84,7 \pm 12,2$ \\
\hline FR (irpm) & $21,2 \pm 1,7$ \\
\hline PAS (mmHg) & $137,0 \pm 15,6$ \\
\hline PAD (mmHg) & $77,0 \pm 8,2$ \\
\hline \multicolumn{2}{|l|}{ Dados clínicos } \\
\hline HAS, n (\%) & 7 (70) \\
\hline DM, n (\%) & $8(80)$ \\
\hline \multicolumn{2}{|l|}{ Medicações } \\
\hline IECA, n (\%) & $6(60)$ \\
\hline Metformina, n (\%) & $6(60)$ \\
\hline
\end{tabular}

IMC: Índice de massa corporal; FC: Frequência cardíaca; FR: Frequência respiratória; PAS: Pressão arterial sistólica; PAD: Pressão arterial diastólica HAS: Hipertensão arterial sistêmica; DM: Diabetes Mellitus; IECA: Inibidor da enzima conversora da angiotensina. Dados apresentados em média e desvio padrão ou frequência.

Constatou-se que três pacientes apresentaram ITB menor que 0,90 na pré-intervenção e cinco no pós-intervenção. A claudicação mostrou-se frequente em nossa amostra, sendo que oito pacientes apresentaram tal sintoma. 
Tabela 2 - Valores do Índice Tornozelo-Braquial pré e pós intervenção com exercício e FES.

\begin{tabular}{|c|c|c|c|c|c|c|c|c|c|c|c|c|}
\hline & \multicolumn{5}{|c|}{ Exercício } & \multicolumn{5}{|c|}{ FES } & \multirow{2}{*}{$\begin{array}{c}* * \mathbf{p} \\
0,179\end{array}$} & \multirow[t]{2}{*}{ TR } \\
\hline & Pré & Pós & Variação & $p^{*}$ & TR & Pré & Pós & Variação & $p^{*}$ & TR & & \\
\hline ITB esquerdo & $0,97 \pm 0,10$ & $0,98 \pm 0,11$ & $0,01 \pm 0,00$ & 0,868 & 0,13 & $1,05 \pm 0,15$ & $1,00 \pm 0,16$ & $0,04 \pm 0,01$ & 0,006 & 1,27 & 0,435 & 0,65 \\
\hline
\end{tabular}

TB: Índice tornozelo-braquial; FES: Estimulação elétrica funcional. TR: Tamanho relativo do efeito. * Comparação entre os valores pré e pós dentro de cada intervenção.

** Comparação entre a variação de cada intervenção. Dados apresentados em média e desvio padrão. Valores significativos para $p<0,05$.

Não foi detectada significância estatística quanto ao ITB entre os momentos pré e pós exercício, tanto em relação ao ITB direito, ITB esquerdo ou geral (Tabela 2), tendo apresentado um tamanho de efeito insignificante, conforme classificação proposta por Cohen ${ }^{15}$ (Tabela 3). Este mesmo comportamento foi observado quando aplicada a FES, no qual o ITB direito, esquerdo e geral não apresentarem alteração significativa (Tabela 2). No entanto, ressalta-se que o tamanho do efeito no ITB direito foi grande, no esquerdo muito grande e no ITB geral, um efeito médio. Ao compararmos a diferença entre os valores pré e pós exercício e FES, não foi constatada diferença significativa, ambas com tamanho do efeito médio.

Tabela 1 - Características da amostra.

\begin{tabular}{lc}
\hline Tamanho relativo do d de Cohen & \\
\hline Efeito Insignificante & $(>-0,15$ e $<0,15)$ \\
Efeito Pequeno & $(>0,15$ e $<0,40)$ \\
Efeito Médio & $(>0,40$ e $<0,75)$ \\
Efeito Grande & $(>0,75$ e $<1,10)$ \\
Efeito Muito Grande & $(>1,10$ e $<1,45)$ \\
Efeito Enorme & $>1,45$ \\
\hline
\end{tabular}

\section{DISCUSSÃO}

Os resultados do presente estudo piloto demonstraram haver maior tendência de redução do ITB, quando aplicado a FES do que quando realizado exercício de planti/dorsiflexão de tornozelos. Apesar de não ter havido resultados estatisticamente significativos, o tamanho do efeito foi maior quando aplicada a FES.

A capacidade de exercício, principalmente aeróbico, altera o balanço do sistema nervoso autonômico pelo aumento do tônus parassimpático e diminuição da atividade simpática, proporcionando melhora no $\mathrm{VO}_{2}$ máximo. No entanto, a obesidade per si modifica a frequência cardíaca de recuperação ( $F C r e c)$, tal como comprovado no estudo realizado na Itália, ${ }^{16} \mathrm{em}$ que foi comparado a dinâmica da FC de obesos treinados e não treinados à de indivíduos com peso normal durante teste ergométrico, concluindo que indivíduos obesos, independente da sua aptidão física, apresentaram um recuperação mais lenta.

Indivíduos portadores de $\mathrm{Cl}$ apresentam redução do fluxo sanguíneo durante o exercício, consequente intolerância e dificuldade ao deambular, podendo com frequência apresentar pausas durante tal prática e, em estados mais graves, dificuldade de sustentar o próprio peso corporal. ${ }^{6,17} \mathrm{Em}$ nosso estudo, oito pacientes apresentaram o Questionário de Edimburgo como positivo, demonstrando que a claudicação mostrou-se prevalente devendo ressaltar que nenhum paciente realizava exer- cício físico regular. Possivelmente esses pacientes eram acometidos por DAP, pois na maioria das vezes tais indivíduos são fisicamente inativos, apresentam baixa capacidade aeróbica e força muscular além de baixos escores nos indicadores de qualidade de vida como demonstrado em estudos anteriores. ${ }^{18,19}$ No entanto, em nosso estudo apenas dois pacientes, dos oito $\operatorname{com~} \mathrm{Cl}$, apresentaram ITB menor que 0,90, indicando DAP.

Deve-se ressaltar que o excesso de peso é um importante fator de risco para o desenvolvimento de DAP e que, em nossa amostra, a média do IMC indicou sobrepeso $\left(>29,7 \mathrm{Kg} / \mathrm{m}^{2}\right)$. O ITB é um método não invasivo e de boa reprodutibilidade para o diagnóstico de DAP, sendo os valores menores que 0,90; um preditor de risco para morbidade e mortalidade coronariana e vascular cerebral, pois metade dos pacientes com DAP possuem sintomas dessas doenças. ${ }^{5}$ Dessa forma, em um estudo realizado no Japão, ${ }^{14}$ os autores ressaltaram que o ITB é geralmente medido em repouso, mas valores pós-exercício aumentam a sensibilidade do teste e pode ser utilizado para identificar doença vascular aterosclerótica. Tais autores formularam a hipótese que a diminuição do ITB no pós-exercício esteja relacionada com a presença de disfunção endotelial.

De acordo com o cálculo do " $\mathrm{d}$ " de Cohen, 15 demostrou que a realização do exercício provocou um efeito pequeno sobre a redução do ITB e a FES, mas, ocasionou um efeito grande no ITB, mostrando que a FES agudamente pode agravar a limitação do fluxo arterial periférico em maior ênfase que o exercício. Dessa forma, alguns autores já demonstraram que o exercício é seguro nesses pacientes, podendo ser influenciado por estímulos mecânicos, como também metabólicos, sendo que as alterações metabólicas na fibra muscular aumentam a força e a massa muscular na proporção do treinamento resistido, dependendo da quantidade de resistência (peso) oferecida em cada repetição bem como do número de repetições em cada sessão de treinamento. ${ }^{20}$

O exercício físico pode provocar redução importante na pressão arterial no período pós-exercício, isto é, a pressão arterial pode permanecer abaixo dos valores pré-exercício por um período de 90 minutos. ${ }^{21}$ Além do exercício, tem-se investigado o efeito da FES sobre a força muscular de indivíduos com doenças cardiovasculares. ${ }^{22-24}$ A FES tem sido proposta como uma terapia adjuvante promissora para potencializar os efeitos do treinamento físico, em pacientes envolvidos em programas de reabilitação cardiovascular e nesse sentido, um estudo avaliou a influência da FES sobre a distância percorrida no Teste de Caminhada de Seis Minutos (TC6) em pacientes com insuficiência cardíaca, demonstrando um grande benefício da eletroestimulação em pacientes de âmbito hospitalar, que foram capazes de realizar um programa de reabilitação resultando 
em aumento da distância percorrida no TC6. ${ }^{25}$

A eletroestimulação crônica ocasiona efeitos importantes sobre a capilarização, bem como, notáveis aumentos na densidade capilar e na perfusão e suprimento de oxigênio, o que contribui para o aumento da capacidade aeróbica oxidativa e da resistência à fadiga dos músculos isquêmicos estimulados, o que beneficia os pacientes com DAP. ${ }^{26}$ Tais resultados levaram os autores $^{26}$ a afirmar que, embora a eletroestimulação crônica com uma série de contrações tetânicas também resulte em aumento na proporção capilar/fibra, isso só ocorre em estágios tardios. No entanto, como nosso estudo observou o efeito agudo, este demonstrou um efeito redutor do ITB, após a aplicação da FES.

Sabe-se ainda que a FES aplicada nos membros inferiores recruta grandes grupos musculares, ativa a bomba venosa dos membros inferiores, facilita o retorno venoso, aumenta o volume sistólico e provoca alterações musculares crônicas que incluem o aumento da resistência muscular, hipertrofia e mudanças histoquímicas. ${ }^{27}$ Outros estudos já demonstraram que sua aplicação tem por objetivo o aumento da força e do volume muscular, da vascularização periférica, da força contrátil cardíaca e do fornecimento de oxigênio aos músculos em atividade. ${ }^{27,28}$

Apesar de ser um estudo piloto, onde provavelmente os resultados não foram significativos estatisticamente, devido ao tamanho amostral, algumas limitações quanto à amostra precisam ser citadas, como a dificuldade no recrutamento e aderência dos pacientes ao protocolo de intervenção e o fato de não ter sido avaliada a resposta da dilatação fluxo-mediada da artéria braquial, que teria possibilitado aferir o real comportamento do fluxo arterial de cada paciente.

\section{CONSIDERACÕ̃ES FINAIS}

Em conclusão, nosso estudo demonstrou que o exercício se mostrou mais seguro que o uso da FES, visto que o efeito agudo provocado pela FES ocasionou redução mais acentuada do ITB após sua aplicação, o que pode indicar o agravamento da obstrução arterial do paciente. Faz-se necessária a continuação do estudo a fim de consolidar os resultados e demonstrar se o uso da FES pode comprometer significativamente a circulação periférica.

\section{REFERÊNCIAS}

1. VI Diretrizes Brasileiras de Hipertensão. Rev Bras Hipertens 2010;95(1):1-51.

2. Paula EA, Paula RB, Costa DM N, Colugnati FAB. Avaliação do risco cardiovascular em hipertensos. Rev Lat Am Enfermagem 2013;21(3):820-827. doi: 10.1590/S010411692013000300023.

3. Alves A, Santos R, Sobrinho E, Rocha S, Loch A. Retinopatia em pacientes hipertensos e/ou diabéticos em uma unidade de saúde da família. Rev Bras Oftalmol 2014;72(2):108-111. doi: 10.5935/0034-7280.20140024.

4. Vaz C, Duarte VM, Santos AR, Valente P, Paúl C, Bastos R, Nogueira C, Loureiro L, Silveira D, Teixeira S, Rego D, Matos A, Almeida R. Doença arterial periférica e qualidade de vida.
Angiol Cir Vasc 2013;9(1):1-7.

5. Neto SS, Nascimento JLM. Doença arterial obstrutiva periférica novas perspectivas de fatores de risco. Rev Para Med 2007;21(2):35-39.

6. Gardner AW, Montgomery P, Killewich LA. Natural history of physical function in older men with intermittent claudication. J Vasc Surg 2004;40(1):73-78. doi: 10.1016/j. jvs.2004.02.010.

7. Júnior LTG, Martim JFV. Índice tornozelo-braquial no diagnóstico da doença arterial aterosclerótica carotídea. Rev Bras Hipertens 2010;17(2):117-118.

8. Maffiuletii NA. Physiological and methodological considerations for the use of neuromuscular electrical stimulation. Eur J Appl Physiol 2010;110(2):223-234. doi: 10.1007/s00421-010-1502-y.

9. Tsang CK, Qi H, Liu LF, Zheng XF. Targeting mammalian target of rapamycin (mTOR) for health and diseases. Drug Discov Today 2007;12(3-4):112-124. doi: 10.1016/j. drudis.2006.12.008.

10. Medeiros AHO, Chalegre ST, Carvalho CC. Eletroestimulação muscular: alternativa de tratamento coadjuvante para pacientes com doença arterial obstrutiva periférica. J Vasc Bras 2007;6(2):152-162. doi: 10.1590/S167754492007000200010.

11. Makdisse M, Neto RN, Chagas ACP, BrasilD, Borges JL, Oliveira A, Gordillo J, Balsalobre G, Crozariol L, Pinho M, Oliveira R, Salles AF. Cross-Cultural Adaptation and Validation of the Brazilian Portuguese Version of the Edinburgh Claudication Questionnaire. Arq Bras Cardiol 2007;88(5):501-506. doi: 10.1590/S0066-782X2007000500001.

12. Aboyans $V$, Michael HC, Abraham P, Matthew AA, Mark AC, Dihem C, Fowkes FGR, Hiatt WR, Jönsson B, Lacroix P, Marin B, McDermott MM, Norgren L, Pande RL, Preux PM, Stoffers $H E$, Treat-Jacobson D. Measurement and Interpretation of the Ankle-Brachial Index: A Scientific Statement From the American Heart Association. Circulation 2012;126(24):28902909. doi: 10.1161/CIR.Ob013e318276fbcb.

13. Kim ES, Wattanakit K, Gornik HL. Using the ankle-brachial index to diagnose peripheral artery disease and assess cardiovascular risk. Cleve Clin J Med 2012;79(9):651-661. doi: 10.3949/ccjm.79a.11154.

14. Sato S, Masami K, Otsuki S, Tanaka S, Nakayama N, Makita S, Koshiyama H, Nohara R. Post-exercise ankle-brachial pressure index demonstrates altered endothelial function in the elderly. Jpn Clin Med 2011;7(2):21-24. doi: 10.4137/ JCM.S7173.

15. Thalheimer W, Cook S. How to calculate effect sizes from published research articles: A simplified methodology. WorkLearning Research. 2002

16. Gondoni LA, Titon AM, Nibbio F, Augello G, Caetani G, Liuzzi A. Heart rate behavior during an exercise stress test in obese patients. Nutr Metab Cardiovasc Dis 2009;19(3):170-176. doi: 10.1016/j.numecd.2008.07.001.

17. Stein R, Hriljac I, Halperin JL, Gustavson SM, Teodorescu $\mathrm{V}$, Olin JW. Limitation of the resting ankle-brachial index in symptomatic patients with peripheral arterial disease. Vasc Med 2006;11(1):29-33. doi: 10.1191/1358863x06vm663oa.

18. Mc Dermott MM, Criqui MH, Greeland P, Guralnik JM, Liu K, Pearce WH, Taylor L, Chan C, Celic L, Woolley C, O'Brien MP, Schneider JR. Leg strength in peripheral arterial disease: associations with disease severity and lower-extremity performance. J Vasc Surg 2004;39(3);523-530. doi: 10.1016/j.jvs.2003.08.038. 
COMPARAÇÃO DO EFEITO AGUDO DO EXERCÍCIO COM A ELETROESTIMULAÇÃO FUNCIONAL SOBREO FLUXO ARTERIAL PERIFÉRICO DE INDÍVIDUOS HIPERTENSOS E DIABÉTICOS: UM ESTUDO PILOTO

19. Grams ST, Damiano AP, Monte FG, Mandelli MB, Carvalho T. Marcha de pacientes com doença arterial obstrutiva periférica e claudicaçãointermitente. RevBrasMed Esporte2009;15(4):255259. doi: 10.1590/S1517-86922009000500004.

20. Bevilaqua-Grossi D, Felicio LR, Leocádio LP. Análise do tempo de resposta reflexa dos músculos estabilizadores patelares em indivíduos com síndrome da dor patelofemural. Rev Bras Fisioter 2008;12(1):26-30. doi: 10.1590/S1413 35552008000100006

21. Negrão CE, Rondon MUPB. Exercício físico, hipertensão e controle barorreflexo da pressão arterial. Rev Bras Hipertens 2001;10(6):89-95.

22. Karavidas A, Driva M, Parissis JT, Farmakis D, Mantzaraki V, Varounis C, Paraskevaidis I, Ikonomidis I, Pirgakis V, Anastasiou-Nana M, Filippatos G. Functional electrical stimulation of peripheral muscles improves endothelial function and clinical and emotional status in heart failure patients with preserved left ventricular ejection fraction. Am Heart $\mathrm{J}$ 2013;166(4):760-770. doi: 10.1016/j.ahj.2013.06.021.

23. Smart NA, Dieberg G, Giallauria F. Functional electrical stimulation for chronic heart failure: a meta-analysis. Int $J$ Cardiol 2013;167(1):80-6. doi: 10.1016/j.ijcard.2011.12.019.

24. Deftereos S, Giannopoulos G, Raisakis K, Kossyvakis C, Kaoukis A, Driva M, Pappas L, Panagopoulou V, Ntzouvara O, Karavidas A, Pyrgakis V, Rentoukas I, Aggeli
C, Stefanadis C. Comparison of muscle functional electrical stimulation to conventional bicycle exercise on endothelium and functional status indices in patients with heart failure. Am J Cardiol 2010;106(11):1621-1625. doi: 10.1016/j. amjcard.2010.07.040.

25. Araújo CJS, Gonçalves FS, Bittencourt HS, Santos NG, Mecca Junior SV, Neves JL, Fernandes AM, Aras Junior R, Reis FJ, Guimarães AC, Rodrigues Junior ES, Carvalho VO. Effects of neuromuscular electrostimulation in patients with heart failure admitted to ward. J Cardiothorac Surg 2012;15(7):7-124. doi: 10.1186/1749-8090-7-124.

26. Tsang GMK, Verde MA, Corvo AJ, Smith FCT, Beck S, Hudlicka O, Sherman CP. Chronic muscle stimulation improves ischaemic muscle performance in patients with peripheral vascular disease. Eur J Vasc Surg 1994;8(4):419422. doi: 10.1016/S0950-821X(05)80960-0.

27. Carvalho DCL, Cliquet Jr A. Investigação das alterações osteometabólicas e cardio-respiratórias ocorridas após o treinamento de marcha sob estimulação elétrica neuromuscular em pacientes etraplégicos. Acta Ortop Bras 2006;14(3):1809-4406.

28. Ferreira LL, Vanderlei LCM, Valenti VE. Estimulação elétrica neuromuscular em pacientes graves em unidade de terapia intensiva: revisão sistemática. Eisntein 2014;12(3):361-365. doi: 10.1590/S1679-45082014RW2955.

Como citar: MAHLE, Djeime et al. Comparação do efeito agudo do exercício com a eletroestimulacão funcional sobre o fluxo arterial periférico de indíviduos hipertensos e diabéticos: um estudo piloto. Cinergis, Santa Cruz do Sul, v. 17, n. 4, out. 2016. ISSN 2177-4005. Disponível em: <https://online.unisc.br/seer/index.php/cinergis/article/view/8055>. Acesso em: 11 out. 2016. doi:http://dx.doi.org/10.17058/cinergis.v17i4.8055. 